\title{
A convenient synthesis of new NSAID esters containing amino acid, urea and amide moieties
}

\author{
IVANA PERKOVIĆ \\ ZRINKA RAJIĆ DŽOLIĆ \\ BRANKA ZORC* \\ University of Zagreb \\ Faculty of Pharmacy and Biochemistry \\ 10000 Zagreb, Croatia
}

Accepted March 12, 2013

\begin{abstract}
A convenient synthetic method for the preparation of novel NSAID twin esters $\mathbf{6 a - i}$ containing amino acid residue, urea and amide moieties has been developed. The synthetic pathway applied for the preparation of target compounds and key intermediates 1-benzotriazolecarboxylic acid chloride (1), NSAID benzotriazolides $\mathbf{2} a-\mathbf{c}$ and $N$-(1-benzotriazolecarbonyl)-amino acids 3a-d involved benzotriazole as a synthetic auxiliary. The final preparation step of esters $\mathbf{6 a}-\mathbf{i}$ included the solvent-free reaction of compounds $2 \mathrm{a}-\mathrm{c}$ with amino acid derivatives $5 \mathbf{a}-\mathbf{g}$, bearing two hydroxyl groups, one at each terminal, beside urea and amide functionalities.
\end{abstract}

Keywords: NSAID, ester, amino acid, benzotriazole, urea, amide

Nonsteroidal anti-inflammatory drugs (NSAIDs) belong to a class of drugs used worldwide for the treatment of pain, fever and inflammatory diseases. Relatively new findings indicating that long-term use of NSAIDs is associated with lower risk of some types of cancer $(1,2)$ initiated a growing interest in this class of drugs. Numerous NSAID derivatives have been prepared in order to improve analgesic/anti-inflammatory activity, minimize side effects, prolong plasma half-life, increase COX1/COX2 selectivity, cytostatic activity and change water solubility or lipophilicity (3-5). However, our research is directed towards NSAID prodrugs and NSAID derivatives with dual cyclooxygenase/ 5-lipoxygenase inhibition or potential antioxidative and cytostatic activity. In this light, we have prepared and pharmacologically evaluated numerous amides $(6,7)$, phosphoramidate derivatives (8), semicarbazides and hydroxycarbamoylcarbazides (9), hydroxamic acid NSAID derivatives $(10,11)$ and polymer-NSAID conjugates $(12,13)$. Here, we describe syntheses of novel NSAID esters, derivatives of ibuprofen, ketoprofen and diclofenac, containing amino acid residue, urea and amide moieties, as potential NSAID prodrugs.

\footnotetext{
* Correspondence; e-mail: bzorc@pharma.hr
} 


\section{EXPERIMENTAL}

\section{Materials and methods}

IR spectra were recorded on a FTIR Perkin Elmer Paragon 500 spectrometer (Perkin-Elmer, UK). ${ }^{1} \mathrm{H}$ and ${ }^{13} \mathrm{C}$ NMR spectra were recorded on a Varian Gemini 300 spectrometer (Varian, USA), operating at 300 and $75.5 \mathrm{MHz}$ for ${ }^{1} \mathrm{H}$ and ${ }^{13} \mathrm{C}$ nuclei, respectively. Samples were measured in DMSO- $d_{6}$ solutions at $20^{\circ} \mathrm{C}$ in $5-\mathrm{mm}$ NMR tubes. Chemical shifts $(\delta)$ in ppm were referred to TMS. Elemental analyses were performed on a CHNS LECO analyzer (LECO Corporation, USA). Solvent systems cyclohexane/ethyl acetate (1:1), cyclohexane/ethyl acetate/methanol (3:1:0.5), ethyl acetate/cyclohexane/methanol (3:1:0.1) and dichloromethane/methanol (9.5:0.5 and 9:1) and precoated Merck silica gel $60 \mathrm{~F}_{254}$ plates (Merck, Germany) were used for thin-layer chromatography (TLC). Spots were visualized by short-wave UV light and iodine vapour. Column chromatography was performed on silica gel $(0.063-0.200 \mathrm{~mm})$ (Kemika, Croatia), with dichloromethane/methanol (9.5:0.5), cyclohexane/ethyl acetate/methanol (3:1:0.5) and cyclohexane/ethyl acetate (1:1) as eluents. Benzotriazole $(\mathrm{BtH})$, triphosgene, 2-aminoethanol (ethanolamine), 3-aminopropanol, 5-aminopenthanol and triethylamine (TEA) were purchased from Aldrich (USA). Amino acids were purchased from Kemika, while ibuprofen, ketoprofen and diclofenac were from Pliva and Belupo (Croatia).

Benzotriazole carboxylic acid chloride (1) (14), ibuprofen-benzotriazolide (2a), ketoprofen-benzotriazolide (2b), diclofenac-benzotriazolide (2c) (12), $N$-(1-benzotriazolecarbonyl)-L-alanine (3a), $N$-(1-benzotriazolecarbonyl)-L-leucine (3b), $N$-(1-benzotriazolecarbonyl)-D-phenylglycine (3c), $N$-(1-benzotriazolecarbonyl)-L-phenylalanine (3d) (15) and L-alanine/3-aminopropanol (5a), L-leucine/3-aminopropanol (5b), L-leucine/5-aminopenthanol (5c), D-phenylglycine/2-aminoethanol (5d), D-phenylglycine/5-aminopenthanol (5e), L-phenylalanine/2-aminoethanol (5f), L-phenylalanine/3-aminopropanol $(5 \mathrm{~g})$ ureidoamides (9) were prepared according to our procedures published earlier.

\section{Syntheses of compounds $6 a-i$. General procedure}

A solvent free mixture prepared from NSAID benzotriazolide $2 \mathrm{a}-\mathrm{c}(1 \mathrm{mmol})$, the corresponding ureidoamide $5 \mathrm{a}-\mathrm{g}(0.5 \mathrm{mmol})$ and TEA $(0.202 \mathrm{~g}, 2 \mathrm{mmol})$ was heated until melting occurred $\left(100-125^{\circ} \mathrm{C}\right)$ and kept at that temperature for 15 minutes. The reaction mixture prepared was cooled, dissolved in $30 \mathrm{~mL}$ ethyl acetate and extracted three times with sodium hydroxide solution $(3 \times 30 \mathrm{~mL}$ of water and 10 drops of $5 \% \mathrm{NaOH})$, washed with water, dried over anhydrous sodium sulphate, filtered and evaporated under reduced pressure. Crude products were purified by column chromatography. Analytical and spectral data of synthesized esters $\mathbf{6 a - i}$ are given in Tables I and II.

\section{2-(4-Isobutyl-phenyl)-propionic acid 5-[2-(3-/5-[2-(4-isobutyl-phenyl)- -propionyloxy]-pentyl/-ureido-2-phenyl-acetylamino]-pentyl ester (6a)}

A mixture prepared from $0.307 \mathrm{~g}$ ibuprofen derivative (2a), $0.181 \mathrm{~g}$ D-phenylglycine 5-aminopenthanol ureidoamide (5e) and TEA was heated at $100{ }^{\circ} \mathrm{C}$. Dichloromethane/ methanol 9.5:0.5 was used as eluent for column chromatography. 
2-(4-Isobutyl-phenyl)-propionic acid 3-[2-(3-/3-[2-(4-isobutyl-phenyl)-propionyloxy]-propyl/-ureido-3-phenyl-propionylamino]-propyl ester (6b)

A mixture prepared from $0.307 \mathrm{~g}$ ibuprofen derivative (2a), $0.162 \mathrm{~g}$ L-phenylalanine 3-aminopropanol ureidoamide (5g) and TEA was heated at $100{ }^{\circ} \mathrm{C}$. Dichloromethane/ methanol 9.5:0.5 was used for column chromatography.

\section{2-(3-Benzoyl-phenyl)-propionic acid 3-[2-(3-/3-[2-(3-benzoyl-phenyl)- -propionyloxy]-propyl/-ureido)-propionylamino]-propyl ester (6c)}

Ketoprofen derivative $(\mathbf{2 b}, 0.355 \mathrm{~g})$ in a mixture with L-alanine 3-aminopropanol ureidoamide (5a, $0.124 \mathrm{~g}$ ) and TEA was heated at $125^{\circ} \mathrm{C}$. Dichloromethane/methanol 9.5:0.5 was used for column chromatography.

\section{2-(3-Benzoyl-phenyl)-propionic acid 3-[2-(3-/3-[2-(3-benzoyl-phenyl)-propionyloxy]-} -propyl/-ureido)-4-methyl-pentanoylamino]-propyl ester (6d)

Ketoprofen derivative $(\mathbf{2} \mathbf{b}, 0.355 \mathrm{~g})$ was mixed with L-leucine 3-aminopropanol ureidoamide $(5 \mathbf{b}, 0.145 \mathrm{~g})$ and TEA. The mixture was heated at $100{ }^{\circ} \mathrm{C}$. Cyclohexane/ethyl acetate 1:1 was used as eluent for column chromatography.

2-(3-Benzoyl-phenyl)-propionic acid 5-[2-(3-/5-[2-(3-benzoyl-phenyl)-propionyloxy]-pentyl/-ureido)-4-methyl-pentanoylamino]-pentyl ester (6e)

The titled compound was prepared from a mixture of $0.355 \mathrm{~g}$ ketoprofen derivative (2b), $0.173 \mathrm{~g}$ L-leucine 5-aminopenthanol ureidoamide (5c) and TEA heated at $100{ }^{\circ} \mathrm{C}$. Dichloromethane/methanol 9.5:0.5 was used for purification of the crude product by column chromatography.

\section{2-(3-Benzoyl-phenyl)-propionic acid 2-[2-(3-/2-[2-(3-benzoyl-phenyl)-propionyloxy]-} -ethyl/-ureido)-2-phenyl-acetylamino]-ethyl ester (6f)

To synthesize the title compound, a mixture of $0.355 \mathrm{~g}$ ketoprofen derivative (2b), 0.141 g D-phenylglycine 2-aminoethanol ureidoamide (5d) and TEA was heated at 115 ${ }^{\circ} \mathrm{C}$. Ethyl acetate/cyclohexane/methanol 3:1:0.1 was used as eluent for purification of the crude product by column chromatography.

2-(3-Benzoyl-phenyl)-propionic acid 2-[2-(3-/2-[2-(3-benzoyl-phenyl)-propionyloxy]-ethyl/-ureido)-3-phenyl-propionylamino]-ethyl ester (6g)

A mixture prepared from $0.355 \mathrm{~g}$ ketoprofen derivative (2b), $0.148 \mathrm{~g}$ L-phenylalanine 2-aminoethanol ureidoamide (5f) and TEA was heated at $100{ }^{\circ} \mathrm{C}$. Ethyl acetate/ cyclohexane/methanol 3:1:0.1 was used for column chromatography purification. 
I. Perković et al:: A convenient synthesis of new NSAID esters containing amino acid, urea and amide moieties, Acta Pharm. 63 (2013) $409-418$.

Table I. Analytical and spectral data of compounds $6 a-i$

\begin{tabular}{|c|c|c|c|c|c|c|}
\hline \multirow{2}{*}{ 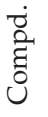 } & \multirow{2}{*}{$\begin{array}{c}\text { Yield } \\
(\%)\end{array}$} & \multirow{2}{*}{$\begin{array}{l}\text { IR (film): } v_{\max } \\
\quad\left(\mathrm{cm}^{-1}\right)\end{array}$} & \multirow{2}{*}{$\begin{array}{l}\text { Molecular } \\
\text { formula } \\
\left(M_{\mathrm{r}}\right)\end{array}$} & \multicolumn{3}{|c|}{ Analysis (calcd./found) (\%) } \\
\hline & & & & C & $\mathrm{H}$ & $\mathrm{N}$ \\
\hline $6 a$ & 80 & $\begin{array}{c}3302,2915,1732,1650,1570,1460 \\
1378,1365,1265,1173,735\end{array}$ & $\begin{array}{l}\mathrm{C}_{45} \mathrm{H}_{63} \mathrm{~N}_{3} \mathrm{O}_{6} \\
(742.00)\end{array}$ & $72.84 / 72.92$ & $8.56 / 8.27$ & $5.66 / 5.95$ \\
\hline $6 b$ & 74 & $\begin{array}{c}3300,2916,1732,1644,1568,1464 \\
1378,1366,1262,1168,748\end{array}$ & $\begin{array}{l}\mathrm{C}_{42} \mathrm{H}_{57} \mathrm{~N}_{3} \mathrm{O}_{6} \\
(699.92)\end{array}$ & $72.07 / 72.56$ & $8.21 / 8.05$ & $6.00 / 5.55$ \\
\hline $6 c$ & 82 & $\begin{array}{c}3302,3065,2927,2869,1731,1659 \\
1635,1569,1556,1449,1380,1318 \\
1284,1250,1206,1173,1079,960 \\
719,644\end{array}$ & $\begin{array}{l}\mathrm{C}_{42} \mathrm{H}_{45} \mathrm{~N}_{3} \mathrm{O}_{8} \\
\quad(719.82)\end{array}$ & $70.08 / 69.77$ & $6.30 / 5.94$ & $5.84 / 5.95$ \\
\hline $6 \mathrm{~d}$ & 90 & $\begin{array}{c}3300,3060,2950,1736,1650,1558 \\
1448,1378,1284,1176,1076,954 \\
704,642\end{array}$ & $\begin{array}{c}\mathrm{C}_{46} \mathrm{H}_{55} \mathrm{~N}_{3} \mathrm{O}_{8} \\
(777.94)\end{array}$ & $71.02 / 70.79$ & $7.13 / 7.00$ & $5.40 / 5.75$ \\
\hline $6 e$ & 73 & $\begin{array}{c}3291,3064,2932,2869,1732,1659 \\
1633,1566,1556,1448,1378,1318 \\
1283,1206,1176,1077,954,721 \\
706,643\end{array}$ & $\begin{array}{c}\mathrm{C}_{49} \mathrm{H}_{59} \mathrm{~N}_{3} \mathrm{O}_{8} \\
(818.01)\end{array}$ & $71.95 / 72.16$ & $7.27 / 7.54$ & $5.14 / 5.44$ \\
\hline $6 f$ & 65 & $\begin{array}{l}3296,3064,2980,1735,1633,1564 \\
1449,1374,1284,1172,1077,698,643\end{array}$ & $\begin{array}{l}\mathrm{C}_{45} \mathrm{H}_{43} \mathrm{~N}_{3} \mathrm{O}_{8} \\
\quad(753.84)\end{array}$ & $71.70 / 72.01$ & $5.75 / 5.34$ & $5.57 / 5.50$ \\
\hline $6 \mathrm{~g}$ & 79 & $\begin{array}{l}3302,3063,2979,2938,1732,1663 \\
1646,1569,1554,1451,1380,1284 \\
1205,1177,1078,956,823,722,644\end{array}$ & $\begin{array}{l}\mathrm{C}_{46} \mathrm{H}_{45} \mathrm{~N}_{3} \mathrm{O}_{8} \\
\quad(767.86)\end{array}$ & $71.95 / 72.31$ & $5.91 / 6.04$ & $5.47 / 5.78$ \\
\hline $6 \mathrm{~h}$ & 68 & $\begin{array}{c}3300,3050,2940,1736,1654,1560 \\
1448,1282,1166,1076,954,700,642\end{array}$ & $\begin{array}{l}\mathrm{C}_{48} \mathrm{H}_{49} \mathrm{~N}_{3} \mathrm{O}_{8} \\
\quad(795.92)\end{array}$ & $72.43 / 71.99$ & $6.21 / 6.42$ & $5.28 / 5.66$ \\
\hline $6 i$ & 77 & $\begin{array}{c}3300,3090,2850,1718,1650,1566 \\
1508,1452,1238,1142,1092,775 \\
744,700\end{array}$ & $\begin{array}{l}\mathrm{C}_{44} \mathrm{H}_{43} \mathrm{Cl}_{4} \mathrm{~N}_{5} \mathrm{O}_{6} \\
(879.65)\end{array}$ & $60.08 / 60.41$ & $4.93 / 4.52$ & $7.96 / 7.61$ \\
\hline
\end{tabular}

2-(3-Benzoyl-phenyl)-propionic acid 3-[2-(3-/3-[2-(3-benzoyl-phenyl)-propionyloxy]-propyl-ureido)-phenyl-propionylamino]-propyl ester (6h)

Ketoprofen derivative $(\mathbf{2 b}, 0.355 \mathrm{~g})$ was mixed with $0.162 \mathrm{~g}$ L-phenylalanine 3-aminopropanol ureidoamide $(5 \mathrm{~g})$ and TEA. The mixture was heated at $120{ }^{\circ} \mathrm{C}$. The crude product was purified on the chromatographic column using ethyl acetate/cyclohexane/ methanol 3:1:0.1 as eluent.

[2-(2,6-Dichloro-phenylamino)-phenyl]-acetic acid 3-/2-[3-(3-/2-[2-(2,6-dichloro-phenylamino)-phenyl]-acetoxy/-propyl)-ureido]-3-phenyl-propionylamino/-propyl ester (6i)

A mixture of $0.397 \mathrm{~g}$ diclofenac derivative (2c), $0.162 \mathrm{~g}$ L-phenylalanine 3-aminopropanol ureidoamide $(5 \mathrm{~g})$ and TEA was heated at $100{ }^{\circ} \mathrm{C}$. Column chromatography with dichloromethane/methanol 9.5:0.5 as eluent was used for purification of the crude product. 


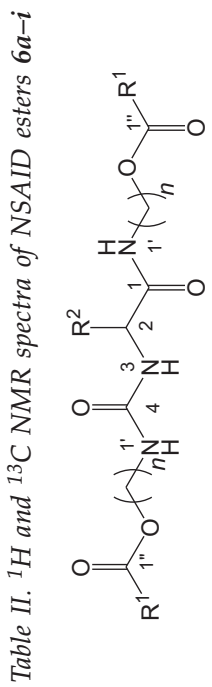

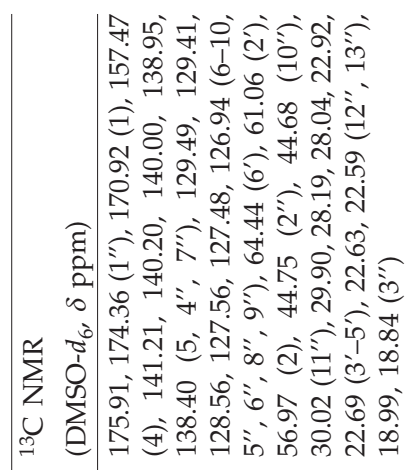

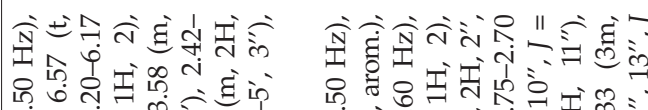

ம

में के

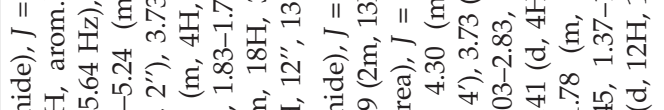

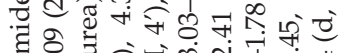

ڤิ

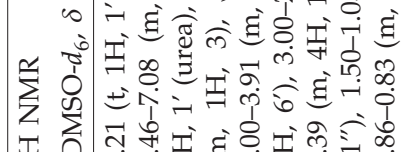

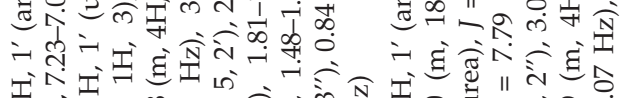

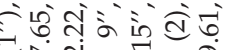

चले

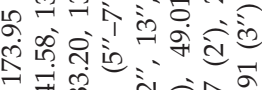

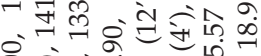

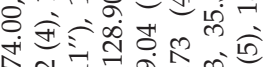

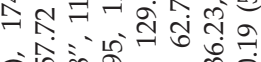

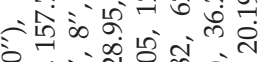

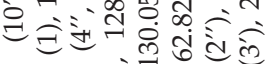

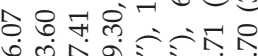

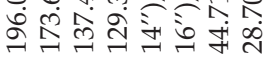

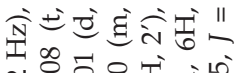

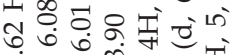
ம் =

के तु $\hat{\mathbb{N}}$ ते के

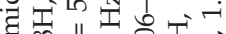
ब्च

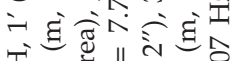

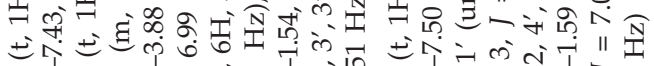
б

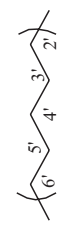

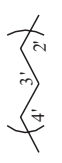

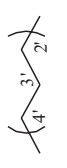<smiles>c1ccc2ccccc2c1</smiles><smiles>CCCc1ccccc1</smiles>

ت<smiles>CC(C)Cc1ccc(C(C)C)cc1</smiles>

ฮ

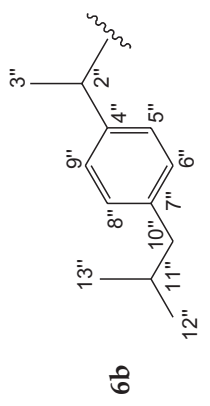




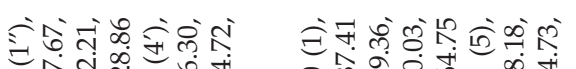

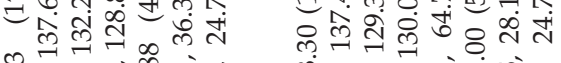

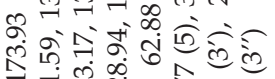

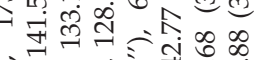

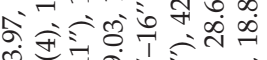

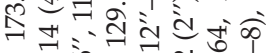

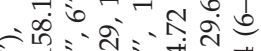
है? ड़े 8 \&

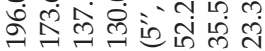

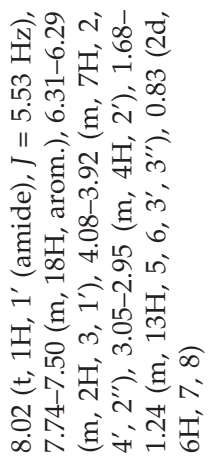
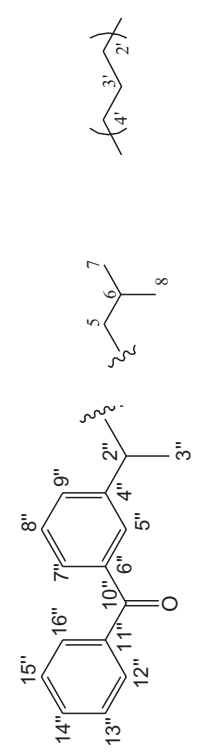

ఫ

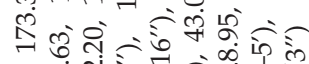

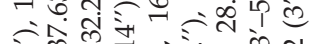

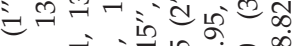

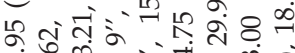
एँ

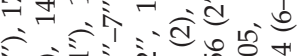

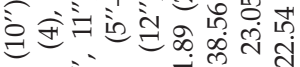

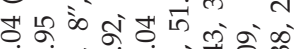
ڤั

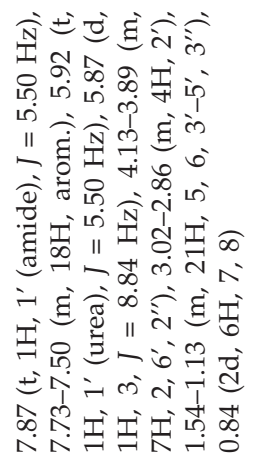

$$
\text { रे }
$$

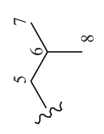<smiles>CC(C)=C1C=CC=C(C(=O)c2ccccc2)C1</smiles>

\&

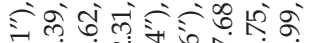

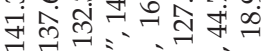
एं के के

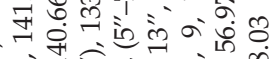

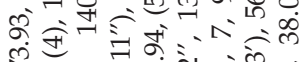

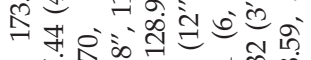

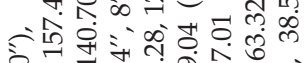

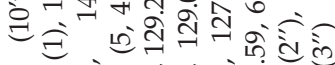

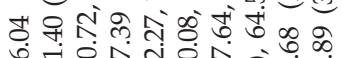
ڤ청

ิㅗํำ-

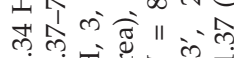
+盗式的

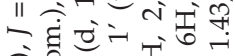
苛 武式式界 ฮ્ટ

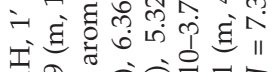

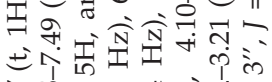

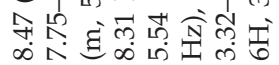

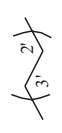

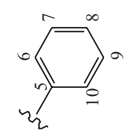<smiles>CC(C)=C1CCCC(C(=O)c2ccccc2)C1</smiles>

$\breve{b}$ 


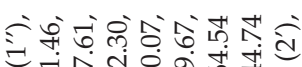
荘全

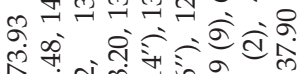

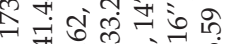

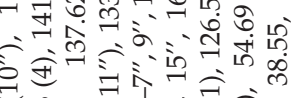

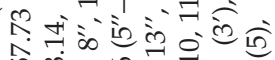

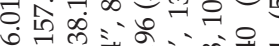

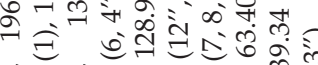

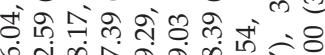

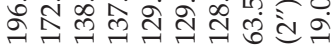

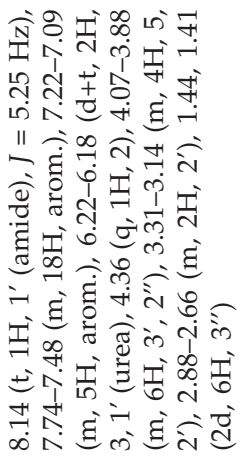
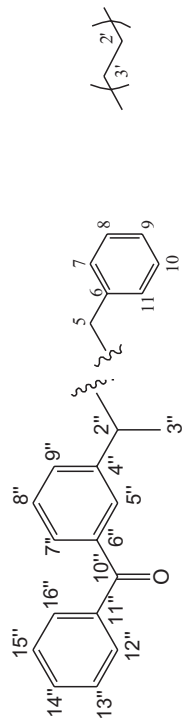

so

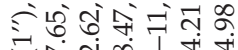
تصن मेन लिख

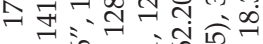

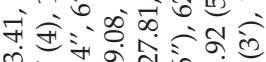

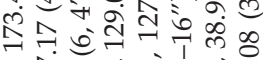
= לे 员

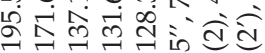

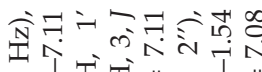

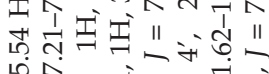

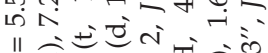
입.

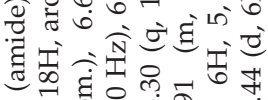

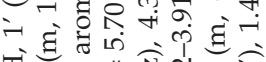

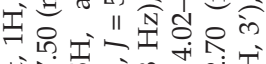

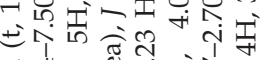

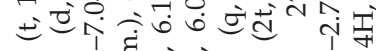

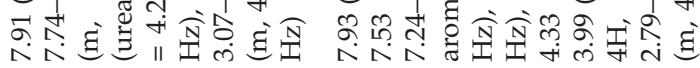
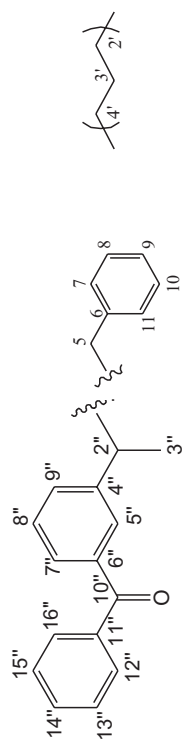

항

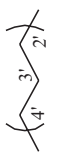

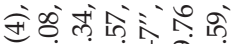

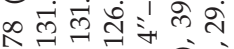

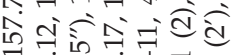

$=\vec{m}=-i d \infty$ क⿺⿻一𠃋十

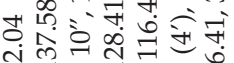
N-1.-

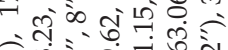
का iो करते

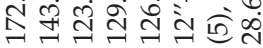

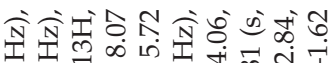

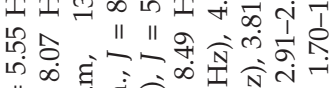

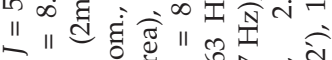
के

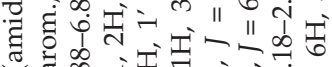

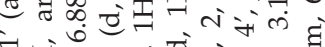
+ீं

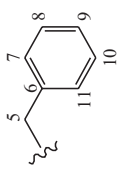<smiles>CCNc1cccc2cccc(O)c12</smiles>

7

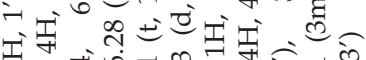




\section{RESULTS AND DISCUSSION}

Synthesis of novel NSAID twin esters $6 \mathbf{a}-\mathbf{i}$ containing amino acid residue, urea and amide moieties is described. The synthetic pathway applied for the preparation of target compounds is presented in Scheme 1. The key intermediates 1-benzotriazolecarboxylic acid chloride (1), NSAID benzotriazolides 2a-c, $N$-(1-benzotriazolecarbonyl)-amino acids $3 \mathbf{a}-\mathbf{d}$ and ureidoamides $5 \mathbf{a}-\mathbf{g}$ were prepared according to our procedures reported elsewhere $(9,12,14,15)$. 1-Benzotriazolecarboxylic acid chloride (1) was synthesized from benzotriazole and triphosgene (14) and further used in the preparation of $N$-(1-benzotriazolecarbonyl)-amino acids 3a-d (15). Two aliphatic (L-alanine, L-leucine) and two aromatic (D-phenylglycine, L-phenylalanine) amino acids were used in experiments. The prepared amino acid derivatives $3 \mathbf{a}-\mathbf{d}$ were transformed to the corresponding chlorides $4 \mathbf{a}-\mathbf{d}$ by means of thionyl chloride. In the reaction with aminoalcohols (2-aminoethanol, 3-aminopropanol or 5-aminopenthanol), they gave ureidoamides 5a-g (9). 1-Benzotriazolecarboxylic acid chloride (1) was also used in the preparation of ibuprofen (2a), ketoprofen (2b) and diclofenac benzotriazolide (2c) (12). The benzotriazole moiety in compounds $2 \mathrm{a}-\mathbf{c}$ activated the nucleophilic substitution of molecules with hydroxyl groups present in ureido amides $5 \mathbf{a}-\mathbf{g}$, allowing preparation of the final esters $\mathbf{6 a} \mathbf{a}-\mathbf{i}$.

Structures of compounds $6 \mathrm{a}-\mathbf{i}$ were deduced from analyses of their IR, ${ }^{1} \mathrm{H}$ and ${ }^{13} \mathrm{C}$ NMR spectra and were confirmed by elemental analysis (Table I). Chemical shifts were consistent with the proposed structures of novel compounds. IR spectra of esters $6 \mathbf{a}-\mathbf{i}$ showed characteristic bands for $\mathrm{NH}$ at $3302-3291 \mathrm{~cm}^{-1}$ and three different carbonyl groups at 1736-1718, 1663-1633 and 1570-1556 $\mathrm{cm}^{-1}$, respectively. In ${ }^{1} \mathrm{H}$ NMR spectra, carbamide

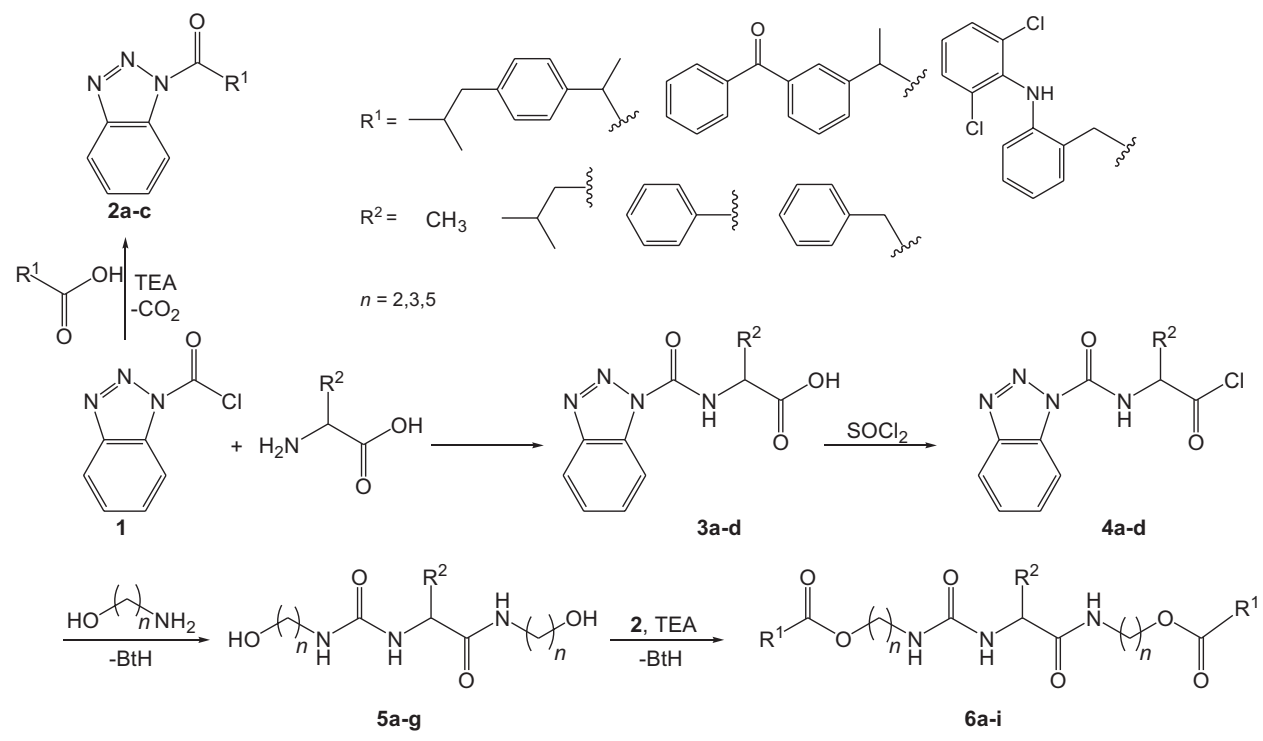


NH-1' appeared as triplets between 6.61 and $5.92 \mathrm{ppm}$, while the NH-3 proton appeared as a doublet between 6.84 and $5.87 \mathrm{ppm}$. Amide $\mathrm{NH}-1^{\prime}$ appeared as a triplet between 8.47 and $7.87 \mathrm{ppm}$. All three $\mathrm{NH}$ groups were exchangeable with $\mathrm{D}_{2} \mathrm{O}$. Ester carbonyls $\mathrm{C}-1$ « in ${ }^{13} \mathrm{C}$ NMR spectra appeared as only one or two very close signals between 175.91 and 172.09 ppm. Amide C-1 atom was located between 173.67 and 170.92 ppm, while urea carbonyl C-4 appeared between 158.14 and 157.17 ppm. Ketoprofen derivatives 6c-h showed additional carbonyl C-10« uniformly located at $196 \mathrm{ppm}$. Detailed NMR data of all new compounds are given in Table II.

\section{CONCLUSIONS}

The environmentally friendly solvent-free protocol for synthesis of novel NSAID esters containing amino acid residue, urea and amide moieties has been described. The prepared esters are potential NSAID prodrugs. Their analgesic, anti-inflammatory and antioxidative activities still remain to be evaluated.

Acknowledgements. - This study was supported by the Ministry of Science, Education and Sports of the Republic of Croatia (Project 006-0000000-3216).

\section{REFERENCES}

1. D. Wang and R. N. DuBois, Eicosanoids and cancer, Nat. Rev. Cancer 10 (2010) 181-193; DOI: $10.1038 /$ nrc2809.

2. E. R. Rayburn, S. J. Ezell and R. Zhang, Anti-inflammatory agents for cancer therapy, Mol. Cell. Pharmacol. 1 (2009) 29-43.

3. B. Zawidlak-Wegrzynska, M. Kawalec, I. Bosek, M. Luczyk-Juzwa, G. Adamus, A. Rusin, P. Filipczak, M. Glowala-Kosinska, K. Wolanska, Z. Krawczyk and P. Kurcok, Synthesis and antiproliferative properties of ibuprofen-oligo(3-hydroxybutyrate) conjugates, Eur. J. Med. Chem. 45 (2010) 1833-1842; DOI: 10.1016/j.ejmech.2010.01.020.

4. S. Fogli, I. Banti, F. Stefanelli, L. Picchianti, M. Digiacomo, M. Macchia, M. C. Breschi and A. Lapucci, Therapeutic potential of sulindac hydroxamic acid against human pancreatic and colonic cancer cells, Eur. J. Med. Chem. 45 (2010) 5100-5107; DOI: 10.1016/j.ejmech.2010.08.019.

5. W. Liu, J. Zhou, K. Bensdorf, H. Zhang, H. Liu, Y. Wang, H. Qian, Y. Zhang, A. Wellner, G. Rubner, W. Huang, C. Guo and R. Gust, Investigations on cytotoxicity and anti-inflammatory potency of licofelone derivatives, Eur. J. Med. Chem. 46 (2011) 907-913; DOI:10.1016/j.ejmech.2011. 01.002 .

6. Z. Rajić, D. Hadjipavlou-Litina, E. Pontiki, M. Kralj, L. Šuman and B. Zorc, The novel ketoprofen amides - Synthesis and biological evaluation as antioxidants, lipoxygenase inhibitors and cytostatic agents, Chem. Biol. Drug Des. 75 (2010) 641-652; DOI: 10.1111/j.1747-0285.2010.00963.x.

7. Z. Rajić, D. Hadjipavlou-Litina, E. Pontiki, J. Balzarini and B. Zorc, The novel amidocarbamate derivatives of ketoprofen: Synthesis and biological activity, Med. Chem. Res. 20 (2010) 210-219; DOI: $10.1007 / \mathrm{s} 00044-010-9309-2$.

8. K. Wittine, K. Benci, Z. Rajić, B. Zorc, M. Kralj, M. Marjanović, K. Pavelić, E. De Clercq, G. Andrei, R. Snoeck, J. Balzarini and M. Mintas, The novel phosphoramidate prodrugs of NSAID 3-hydroxypropylamides: Synthesis, cytostatic and antiviral activity evaluations, Eur. J. Med. Chem. 44 (2009) 143-151; DOI: 10.1016/j.ejmech.2008.03.037. 
9. I. Perković, I. Butula, M. Kralj, I. Martin-Kleiner, J. Balzarini, D. Hadjipavlou-Litina, A-M. Katsori and B. Zorc, Novel NSAID 1-acyl-4-cycloalkyl/arylsemicarbazides and 1-acyl-5-benzyloxy/ hydroxy)carbamoylcarbazides as potential anticancer agents and antioxidants, Eur. J. Med. Chem. 51 (2012) 227-238; DOI: 10.1016/j.ejmch.2012.02.046.

10. Z. Rajić, I. Butula, B. Zorc, S. Kraljević Pavelić, K. Hock, K. Pavelić, L. Naesens, E. De Clercq, J. Balzarini, M. Przyborowska, T. Ossowski and M. Mintas, Cytostatic and antiviral evaluations of NSAID hydroxamic acids, Chem. Biol. Drug Des. 73 (2009) 328-338; DOI: 10.1111/j.1747-0285. 2009.00774x.

11. Z. Rajić, I. Perković, I. Butula, B. Zorc, D. Hadjipavlou-Litina, E. Pontiki, S. Pepeljnjak and I. Kosalec, Synthesis and biological evaluation of O-methyl and $O$-ethyl NSAID hydroxamic acids, J. Enzyme Inhib. Med. Chem. 24 (2009) 1179-1187; DOI: 10.1080/14756360902779128.

12. B. Zorc, S. Antolić and I. Butula, Macromolecular prodrugs. I. Synthesis of some non-steroidal anti-inflammatory drug esters, Acta Pharm. 43 (1993) 127-133.

13. M. Barbarić, M. Kralj, M. Marjanović, I. Husnjak, K. Pavelić, J. Filipović-Grčić, D. Zorc and B. Zorc, Synthesis and in vitro antitumor effect of diclofenac and fenoprofen thiolated and nonthiolated polyaspartamide-drug conjugates, Eur. J. Med. Chem. 42 (2007) 20-29; DOI: 10.1016/ j.ejmech.2006.08.009.

14. I. Kalčić, M. Zovko, M. Jadrijević-Mladar Takač, B. Zorc and I. Butula, Synthesis and reactions of some azolecarboxylic acid derivatives, Croat. Chem. Acta 76 (2003) 217-228.

15. I. Butula, B. Zorc and V. Vela, Reaktionen mit 1-Benzotriazol carbonsaurechlorid.VII. Die Umsetzung mit Aminosauren, Croat. Chem. Acta 54 (1981) 435-440. 